\title{
Measurements of Turbulent Swirl Flame Dynamics in an Ethylene-fuelled Gas Turbine Model Combustor at Elevated Pressure
}

\author{
Isaac G. Boxx ${ }^{1}$, Klaus-Peter Geigle ${ }^{2}$ and Wolfgang Meier. ${ }^{3}$ \\ German Aerospace Center (DLR), Stuttgart. Germany \\ Campbell D. Carter ${ }^{4}$ \\ Air Force Research Laboratory, Wright-Patterson AFB,OH. USA
}

\section{Background}

$\mathrm{I}_{\mathrm{i}}^{\mathrm{N}}$ $\mathrm{N}$ anticipation of increased regulation of soot emissions from aviation engines there has been considerable effort in recent years to better understand, model and predict soot formation in gas turbine combustors. Experimental studies in this effort generally fall into one of two categories: 1) detailed studies of chemical kinetics and mechanisms of specific sub-processes of soot formation, agglomeration and oxidation in simple, well-characterized test flames (e.g., laminar Bunsen or diffusion flames) and 2) studies focusing on system-level parameters such as global soot emissions vs fuel grade and combustor pressure at the expense of detailed understanding of specific subprocesses. Although both categories have specific strengths, the understanding yielded by each is of limited use for the purpose of predictive modelling of gas turbine combustors.

The German Aerospace Center (Deutsches Zentrum für Luft- und Raumfahrt, DLR) has led an effort in recent years to bridge the gap between fundamental scientific studies of soot formation and system-level characterization of gas turbine combustors. This effort has focused on acquiring detailed measurements of a series of soot-generating flames in a generic, swirl-stabilized combustor at elevated pressure. These flames are designed to capture much of the complexity of a modern, swirl-stabilized gas turbine combustor, while maintaining excellent optical access for point- and planar laser measurement techniques.

As part of this effort, Lammel et al. (2007) applied laser-induced incandescence (LII) and coherent anti-Stokes Raman scattering (CARS) spectroscopy to quantify the effects of pressure, equivalence ratio and secondary oxidation air on mean soot distribution in swirl-stabilized, sooting ethylene-air flames at pressures up to 9 bars and thermal loads up to $45 \mathrm{~kW}$. Their results showed the highest soot concentrations were to be found in the lower part of the inner recirculation zone (IRZ) of the combustor, where residence times and local fluid temperatures are high. The combustor used by Lammel et al. (2007) was based on the well-characterized DLR Dual-swirl Gas Turbine Model Combustor or "DS-burner" [2,3] applied LII, CARS and particle image velocimetry (PIV) in a similar combustor at atmospheric pressure and found that injection of secondary air downstream of the flame zone results in drastic changes to soot distribution in the combustor. Geigle et al. (2014) extended these measurements (in a slightly modified burner geometry) to include flames at pressures up to 5 bars and observed that, in addition to pressure and global equivalence ratio, soot concentration and distribution were sensitive to the ratio of air flow supplied to the inner- and outer swirl nozzles. Geigle et al. (2015) applied simultaneous LII and planar laser-induced fluorescence of $\mathrm{OH}(\mathrm{OH}-\mathrm{PLIF})$ to study the spatial correlation of soot and high temperature combustion products in these flames. Their results showed the addition of air downstream of the main flame zone resulted in secondary combustion zones that consume the soot (previously observed in the IRZ) or even prevent soot formation in the first place.

These studies produced a rich database of experimental measurements on sooting, swirl-stabilized flames in a gas turbine model combustor. This database, however, is limited to single-shot measurements which yield only mean and fluctuating quantities of interest. Soot formation, agglomeration and oxidation are highly dynamic processes, dependent upon multiple tightly coupled parameters. It is therefore of considerable interest to acquire time-resolved measurements of quantities such as velocity, soot distribution and reaction zone location. Furthermore, these studies have focused primarily upon globally fuel-rich flame conditions. Although this is certainly of key interest in understanding soot-dynamics, it has been observed that even (globally) lean flames can

\footnotetext{
${ }^{1}$ Staff Scientist, Institute of Combustion Technology. Stuttgart, Germany. AIAA Associate Fellow.

${ }^{2}$ Staff Scientist, Institute of Combustion Technology. Stuttgart, Germany.

${ }^{3}$ Head of Laser Diagnostics Division, Institute of Combustion Technology. Stuttgart, Germany. AIAA Member.

${ }^{4}$ Principal Aerospace Engineer, AFRL/RQHF. 1950 Fifth Street, Wright-Patterson AFB. AIAA Associate Fellow.
} 
produce soot, particularly in regions such as the IRZ, where long-residence times and incomplete fuel-air mixing may be present.

The objective of this study is to expand our understanding of the structure and dynamics of turbulent, swirlstabilized flames in the GT model combustor described in Geigle et al. (2015). This was accomplished by acquiring long-duration, simultaneous stereo PIV (sPIV) and OH-PLIF measurements in turbulent, swirl-stabilized ethyleneair flames in this burner at pressures up to 7 bars. As high-bandwidth imaging diagnostics are still a developing technology in the field of high-pressure turbulent combustion, a secondary goal was to characterize the performance and capabilities of high-bandwidth laser measurement systems based on (relatively) low pulse-energy DPSS laser systems.

\section{Experiment Configuration}

The experiment consists of two major components; the burner and the laser measurement system. Each component is described (separately) below.

\section{A. Burner}

The burner used in this study is of the same design as that described in Geigle et al. (2015). It consists of a swirled fuel-air injector and a combustion chamber (with a square cross-section) mounted in an optically-accessible pressure housing. The swirled injector consists of a pair of coaxial nozzles, separated by a ring of fuel-injection channels. The inner nozzle is $12.3 \mathrm{~mm}$ in diameter. The outer (annular) nozzle has an inner and outer diameter of 14.4 and $19.8 \mathrm{~mm}$, respectively. Air for the central and annular nozzles is supplied through separate pleni. Swirl is induced in both nozzles via a set of radial swirl vanes. Gaseous fuel $\left(\mathrm{C}_{2} \mathrm{H}_{4}\right)$ is injected via a ring of 60 straight channels $\left(0.5 \times 0.4 \mathrm{~mm}^{2}\right)$ located between the central and the annular nozzles. This is designed to mimic the atomizing lip between swirled air flows in liquid-fuelled gas turbine injectors. The exit planes of the fuel and air flows are located at the level of the combustion chamber inlet (defined as height $h=0 \mathrm{~mm}$ ), ensuring maximal optical access to flame at the nozzle exit.

The combustion chamber measures $120 \mathrm{~mm}$ in height and has a square section of $68 \times 68 \mathrm{~mm}^{2}$. Four quartz windows $(127 \mathrm{~mm} \times 59 \mathrm{~mm} \times 3 \mathrm{~mm})$ are mounted between four water-cooled metal posts, yielding an optically accessible area of $51.4 \mathrm{~mm} \times 120 \mathrm{~mm}$. The water-cooled top plate has a cylindrical exhaust hole (diameter=40 mm, length=24 mm), linked to the combustion chamber by a curvature. Each of the four corner posts has an additional air duct of 5-mm diameter for the injection of secondary air into the combustor at a height of $80 \mathrm{~mm}$. This secondary air injection can be used to simulate combustor cooling or the effect of oxidation air on soot formation and agglomeration in the combustor. Pressure fluctuations in the combustor were monitored using a fast response pressure transducer (Kistler Model 4045A20), connected to the exhaust cap via a 4mm (i.d.) stainless steel tube.

The combustor is mounted in a water-cooled, stainless-steel pressure housing with four large windows for optical access $\left(60 \times 120 \mathrm{~mm}^{2}\right)$. The pressure inside the housing is adjusted by partially blocking the exhaust port with a movable piston. An air flow through the gap between the combustion chamber and the pressure housing serves as air cooling for the windows of the combustion chamber. The mean (inner) surface temperature of the windows is estimated to reach $773-1173 \mathrm{~K}$ during burner operation, depending on the position relative to the location of the flame. Although this is well below the melting point of fused silica, the surface temperature is believed to fluctuate above the softening point of the material, dramatically increasing its susceptibility to particulate-fouling. This is a particularly challenging problem for imaging techniques such as PIV, since particle adhesion to the windows will limit experimental run times (with optical measurements) and potentially result in permanent window damage too.

\section{B. Flame Conditions}

Flames were measured at five operating points in this study. These conditions are summarized in Table 1. Operating conditions 1 - 3 were designed to study the effect of increasing pressure and thermal load on a flame of constant stoichiometry and bulk flow velocity. Conditions 2, 4 and 5 were designed to identify the effect of varying flame stoichiometry at constant pressure and bulk flow velocity. In all cases, $70 \%$ of the total air flow was delivered to the combustor via the inner swirl nozzle and 30\% through the outer. In Case 5, additional oxidation air (40\% by volume) was injected downstream of the swirl nozzle in order to safely oxidize unburned fuel remaining in the exhaust gas.

\section{Measurement System}

The combined sPIV/OH-PLIF measuring system used in this study has been described previously in the literature [6,7]. As such, only a brief description is provided here. 


\section{Planar Laser-Induced Fluorescence}

The OH-PLIF system consists of a conventional (albeit modified) frequency-doubled dye laser and an intensified CMOS camera. The dye laser (Sirah Cobra-Stretch HRR, using Rhodamine 6G in ethanol) was pumped with a frequency-doubled, Q-switched, diode-pumped solid state Nd:YLF laser (Edgewave IS-811E). At $3.1 \mathrm{kHz}$, the timeaverage output from the dye laser at $283.2 \mathrm{~nm}$ was $\approx 0.9 \mathrm{~W}$ or $280 \mu \mathrm{J} /$ pulse. The laser line width at $566 \mathrm{~nm}$ was 0.06 $\mathrm{cm}-1$. Wavelength tuning of the dye laser to the peak of the isolated Q1(7) line of the A-X $(1,0)$ band of OH was monitored continuously using a setup consisting of a laminar reference flame and a photomultiplier tube for fluorescence detection.

The 283.2-nm beam was formed into a sheet of $\approx 40 \mathrm{~mm}$ (high in the probe region) using two fused silica lenses $\left(\mathrm{f}_{\mathrm{plif1}}=-50 \mathrm{~mm}, \mathrm{f}_{\text {plif2 }}=150 \mathrm{~mm}\right)$ in a cylindrical telescope configuration and focused to a waist using a third cylindrical lens ( $f_{\text {plif3 }}=750 \mathrm{~mm}$ ). The 283-nm laser sheet was overlapped with the PIV laser sheet using a pair of dichroic mirrors (see Fig. 1). The PIV and PLIF sheets were overlapped in the near and far fields and also in the probe region; this process was checked daily, and the overlap was found to be consistently very good.

Fluorescence signal was acquired with a CMOS camera (LaVision HSS6), external two-stage, lens-coupled intensifier (LaVision HS-IRO) and a fast Cerco $100 \mathrm{~mm}, \mathrm{f} / 2.8$ lens that is AR coated for the UV. Background luminosity was reduced using a 100-ns intensifier gate, and elastic (particle) scattering at $283 \mathrm{~nm}$ was blocked using a high-transmission (> $80 \%$ at $310 \mathrm{~nm}$ ) bandpass interference filter (Laser Components $\mathrm{GmbH}$ ).

\section{Particle Image Velocimetry}

The stereoscopic PIV system is based on a dual-cavity, diode-pumped, solid state Nd:YAG laser (Edgewave, IS$611 \mathrm{DE}$ ) and a pair of CMOS cameras (LaVision HSS8). The laser produces 2.6-mJ pulses at $532 \mathrm{~nm}$ (each $\approx 14 \mathrm{~ns}$ in duration) at a repetition rate up to $10 \mathrm{kHz}$. As the laser is pulse-energy (as opposed to quasi-cw average power) limited, this corresponds to $24.4 \mathrm{~W}$ per head at $9.3 \mathrm{kHz}$. Pulse timing separation for the PIV system was set to $\Delta \mathrm{t}=$ 15 or $20 \mu \mathrm{s}$, depending on the run condition. The OH-PLIF excitation pulses were synchronized to fall on every third PIV measurement cycle.

As with the PLIF system, three cylindrical lenses were used to form the sheet: $f_{\text {piv } 1}=-38 \mathrm{~mm}, \mathrm{f}_{\text {piv } 2}=250 \mathrm{~mm}$, and $\mathrm{f}_{\text {piv3 }}=700 \mathrm{~mm}$. To minimize noise arising from inter-frame particle dropout, the beam waist was located somewhat beyond the probe region. Mie scattering from titanium dioxide $(\mathrm{TiO} 2)$ particles (nominal diameter $\approx 0.5 \mu \mathrm{m}$ ) seeded into the air flow of each nozzle was imaged using a pair of CMOS cameras. Both cameras were mounted on the same side of the laser sheet and had sufficient on-board memory (8 GB) for 8192 dual-frame images (i.e., 0.8 seconds imaging) at $512 \times 640$ pixel resolution. For both PIV cameras, scattered light was collected with a $100-\mathrm{mm}$ focal length, f/2.8 lens (Tokina), and image-blur due to off-axis defocusing was corrected using a Scheimpflug adaptor between the lens and camera. Perspective distortion was corrected using a dual-plane, three dimensional imaging target (LaVision Type 7). The same target was used to map the fields of view of the stereoscopic PIV and PLIF systems to one another. Image mapping, calibration, and particle cross-correlations were completed using a commercial, multi-pass adaptive window offset cross-correlation algorithm (LaVision DaVis 8.2). Final window size and overlap were $24 \times 24$ pixels and 50\%, respectively. This corresponds to a spatial resolution of $2 \mathrm{~mm}$ and vector spacing of $1 \mathrm{~mm}$. Based on the \pm 0.1 pixel uncertainty of the cross-correlation peak-finding algorithm, the random uncertainty of the PIV measurements is estimated to be $\pm 0.41 \mathrm{~m} / \mathrm{s}$.

\section{Results and Discussion}

In this section, the PIV and PLIF results will first be discussed individually, with a focus on achievable measurement quality and a global characterization of the flow-field and flames. After that, phase averaging of the PIV and PLIF measurements with respect to the most energetic modes of the turbulent flow-field will be used to identify the dominant flow-field-flame interaction at each condition and characterize its effect on flame stabilization.

\section{A. PLIF Measurements}

Figure 2 shows a series of OH-PLIF images acquired at $3.1 \mathrm{kHz}$ in lean $(\phi=0.67)$, ethylene-air flames at 3,5 and 7 bars. These images illustrate several interesting characteristics of high-bandwidth measurements. It is clear from Figure 2 that the signal-to-noise ratio (SNR) of the PLIF images decreases significantly with increasing pressure. This is consistent with the deleterious effects of increased molecular collisions with increasing pressure described earlier. Table 2 outlines the peak SNR of the PLIF measurements with increasing pressure. In this case, SNR is defined based on the peak (ensemble-average) signal intensity in an image vs. the root-mean-square of a small square of the background signal levels. 
Figure 2 also illustrates increasing pressure does not result in a uniform decrease in SNR across the image. For example, whereas at 3 bars pressure, the OH-PLIF signal distribution is fairly uniform in the regions of burned gas, at 7 bars one sees a clear drop-off in $\mathrm{OH}$ signal-intensity with increasing distance from the reaction zone. This effect is clearly associated with the increased rate of three-body collisions (the key depletion mechanism for the $\mathrm{OH}$ radical) resulting in less super-equilibrium concentrations of $\mathrm{OH}$ compared to at lower pressure. In addition, images obtained at 7 bars show noticeable beam-steering in the direction of laser propagation (left-to-right, in this case). Although beam-steering certainly occurs at atmospheric pressure, the increased density of the reactants at elevated pressure, together with the increased flame wrinkling associated with increasing $\mathrm{Re}_{\mathrm{t}}$ exacerbates this effect.

The image sequences in Figure 2 also illustrate the effect of increasing chamber pressure on the structure of this flame. For example, at 3 bar we observe relatively smooth interfaces between burned and unburned gasses. Although the slow depletion of $\mathrm{OH}$ radicals (via three-body recombination collisions in post-flame gases) makes it a poor marker of reaction zone location, it has been shown in previous studies [8] that regions of high OH-gradient along the interface of burned and unburned gas in this burner are associated with the reaction zone. The relatively smooth interface between burned and unburned gases in the first row of Figure 2 is therefore indicative of mild flame wrinkling and little, if any, break-up or fragmentation of the flame zone. The measurements acquired at 5 bars (shown in Row 2) show more wrinkled reaction zones than those acquired at 3 bars. This is consistent with increased $\mathrm{Re}_{\mathrm{t}}$ resulting from the increased chamber pressure. Despite the increased flame wrinkling, the reaction zones at 5 bars show little sign of break-up or fragmentation. The PLIF measurements acquired at 7 bars (shown in Row 3) show similar levels of flame wrinkling to that seen at 5 bars, and also a lack of flame break-up and/or fragmentation. This suggests that for the range of pressures used in this study, increasing $\operatorname{Re}_{t}$ does not adversely affect flame stabilization.

\section{Flame Surface Density}

A key quantity of interest in these measurements is the flame surface density (FSD), a measure of spatial distribution and density of the reaction zone layers. This quantity is derived from the OH-PLIF measurements by identifying the reaction zones in each image (via gradient magnitude filtering) and computing their ensemble average over a large number of frames.

In this study, reaction zones were identified in each image using an image-processing algorithm based on that described in our previous paper [9]. Briefly, this algorithm first corrected each PLIF image for camera sensitivity and laser sheet intensity variations, using a white-field calibration lamp and a mean laser-sheet profile, respectively. The PLIF images are then binned $(2 \times 2)$ to improve SNR and a $5 \times 5$ pixel median filter is applied, followed by an edge-preserving non-linear diffusion (NLD) filter. The gradient-magnitudes of the pre-filtered images are then computed using a Sobel filter, and binarized based on a user-specified threshold. Previous studies have shown that the reaction zones in this burner occur at the interface between burned and unburned gases in the combustor. Therefore, any high-magnitude $\mathrm{OH}$-gradients occurring more than 6 pixels away from a region of unburned reactants (identified by thresholding the corresponding OH-PLIF image according to signal intensity) is discarded. The remaining binary images are morphologically thinned to single-pixel width. Finally, fragments of 10 pixels or less are removed.

Figure 3 shows the mean FSD field for the lean $(\phi=0.67)$ flames from measurements at 3,5 and 7 bars. It is clear from this figure that increasing pressure does not affect the mean flame shape or stabilization point. Figure 3 also illustrates an unusual feature of these flames. Whereas the FSD field shows the presence of a conical flame brush typical of swirl-stabilized flames, this flame brush is augmented by a flame anchored at the outer rim of the swirl nozzle (at $\mathrm{x} \approx+/-10 \mathrm{~mm}$ ). This secondary flame zone appears almost cylindrical, rising vertically from the outer rim of the swirl injector, until it meets and coalesces with the conical flame brush. As the fuel is injected at the inner nozzle (at $\mathrm{x} \approx+/-6 \mathrm{~mm}$ ), it is not clear from these mean FSD plots, how the fuel required to support this secondary flame zone arrives at the outer rim.

Figure 4 shows the mean FSD fields measured at 5 bars pressure, with varying stoichiometry. In contrast with Figure 3, one sees significant variation in mean FSD distribution with increasing global equivalence ratio. Whereas the flame brush shows the same conical shape (supported by the secondary flame anchored at the outer swirl nozzle), the mean FSD along the burner centerline varied significantly with $\phi$. At $\phi=0.45$, which corresponds to near blow-off conditions, FSD is virtually zero distances less than $10 \mathrm{~mm}$ from the exit. Nonetheless, one still sees significant FSD in the secondary flame zone and downstream. As $\phi$ increases, one sees increasing FSD along the burner centerline, as well as in the secondary zone at the outer nozzle. As the mass flow rates of air were varied to maintain a constant bulk flow velocity with increasing pressure, this difference cannot be explained by differences in mean shear from one case to the next. As with the cases shown in Figure 3, it is also not clear how the fuel required to support the secondary flame zone arrives at the outer rim of the swirl nozzle. 


\section{B. PIV Measurements}

\section{Mean Velocity}

Figure 5 shows the mean velocity field for the lean $(\phi=0.67)$ conditions at 3,5 and 7 bars. In this figure, background color corresponds to the axial component velocity, in order to illustrate the location and strength of the inner recirculation zone (IRZ). It is clear from this figure that increasing pressure does not affect the shape of the flowfield or the strength of the recirculation zone. Within the accuracy of the measurement, the mean velocity fields are virtually identical. This observation is consistent with the observation from Figure 3, that increasing pressure did not have a significant effect on the mean flame shape and stabilization point.

Figure 6 shows the mean velocity fields for the flames measured at 5 bars, with varying stoichiometry. As expected (given their identical inlet air flows), the mean velocity fields for the lean $(\phi=0.45$ and 0.67$)$ cases show clear similarity. The mean velocity field of the rich $(\phi=1.2)$ case, however, varies significantly from that of the lean flames. The conical inflow region has a wider opening angle than the lean flames. In addition, at approximately 25 $\mathrm{mm}$ downstream of the nozzle exit, the shape of the IRZ changes significantly. The reason for these differences is not immediately clear, given the identical air flow-rates to the swirl nozzle in each case. The increased opening angle beginning at $25 \mathrm{~mm}$ downstream of the nozzle is likely related to the addition of oxidation air (needed to burn out the excess fuel in the exhaust gas) to the combustor. The difference in shape and strength of the conical inflow region closer to the nozzle, however, is not immediately apparent from the mean velocity fields shown in Figure 6.

\section{Proper Orthogonal Decomposition}

To better understand the mechanisms responsible for the difference in the IRZ and flame shape for the flame conditions shown in Figures 4 and 6, a proper orthogonal decomposition (POD) based analysis was performed on the PIV data. POD is a well-established technique in the field of fluid mechanics [10]. The result of the POD is a set of orthogonal eigenmodes, representing coherent flow structures ordered by their contribution to the turbulent kinetic energy. For each eigenmode, temporal mode coefficients can be obtained by taking the scalar products of the instantaneous flow-fields with the eigenmode. In the present case, the POD was calculated using the method of snapshots [11], based on the first 5000 PIV measurements of each run.

The frequency spectra of the time-series of multiplicative constants for the first eigenmode contain strong peaks at 662,715 and $1081 \mathrm{~Hz}$, for the cases measured at $\phi=0.45,0.67$ and 1.2, respectively. Previous studies on a burner with a very similar geometry $[6,12]$ have shown two dominant modes of oscillation; 1) an axial pulsation, associated with a longitudinal thermo-acoustic mode of the combustor and, 2) a fluid-dynamic structure known as a precessing vortex core. Although those studies focused on flames at atmospheric pressure, it is reasonable to assume the dominant frequencies identified in the POD temporal modes correspond to one or the other of these two modes of oscillation.

To determine the nature of the oscillations identified in the POD, the velocity-field data was phase averaged with respect to time-series of multiplicative constants of the first eigenmode. The phase-averaged data (not presented in this paper) clearly show the dominant flow-field oscillation in the lean flame cases is driven by the axial mode associated with thermoacoustic oscillation. The dominant flow-field oscillation in the rich flame case is clearly associated with the PVC. This finding is consistent with the observations regarding the FSD and mean velocity fields of the corresponding cases. Specifically, the lower mean FSD observed along the burner centerline in the lean flame cases is explained by the axial pulsation translating the flame front periodically toward and away from the injector. The lack of periodic axial translation in the rich case (the PVC primarily forces the flame in a radial direction) explains the higher mean FSD on the burner centerline in that case. The similarity in the mean velocity profiles of the lean cases is also explained by their (identical) inlet mass-flows being forced by the same (axial) mode. The different mean velocity profile observed in the rich case (despite its identical inlet mass flowrate) results from its dominant oscillation being the PVC, which forces the inflow more radially.

\section{Phase-Averaged Data}

Figures 7 and 8 show the FSD distributions of the $\phi=0.67$ and $\phi=1.2$ flames, respectively, phase-averaged with respect to dominant flow-field oscillation identified in the POD. These figures illustrate the physical mechanism responsible for the differences observed in the mean velocity and FSD fields. In Figure 7 one observes that, consistent with the mean FSD fields shown in Figure 4, the flame is constantly anchored at the outer rim of the swirl nozzle $(\mathrm{x} \approx+/-10 \mathrm{~mm})$. The FSD measured in the IRZ, however, varies significantly over the cycle of oscillation. This is explained by the dominant oscillation cycle translating the flame sheet axially along the burner centerline. Figure 7 suggests the axial pulsation, together with the confinement effect of the rectangular combustion chamber, also induces a flow disturbance that transports fuel from its injection ring $(\mathrm{x} \approx+/-6 \mathrm{~mm})$ to sustain the secondary 
flame seen at the outer rim of the injector $(\mathrm{x} \approx+/-10 \mathrm{~mm})$. This observation is supported by noting the significant levels of FSD present at the dump plane in the region $\mathrm{x} \approx+/-(6-10) \mathrm{mm}$ (i.e., across the outer swirl nozzle) at phases $6,7,8$ and 1 .

Figure 8 illustrates the effect of the PVC on the reaction zone in the rich $(\phi=1.2)$ flame. Consistent with the mean FSD fields shown in Figure 6, a secondary flame is constantly anchored at the outer rim of the swirl nozzle (x $\approx+/-10 \mathrm{~mm}$ ). This flame coalesces with the conical flame brush approximately $10 \mathrm{~mm}$ downstream of the dump plane of the combustor. The effect of the PVC is limited primarily to the IRZ. Figure 8 shows the FSD measured in the IRZ is periodically drawn to the left and the right, forced radially as the PVC precesses about the burner centerline. The lack of axial forcing explains the higher FSD (compared to the lean flames) measured in the IRZ and near-nozzle region.

Figures 7 and 8 also illustrate an important feature of high-bandwidth laser imaging diagnostics for the study of flames at elevated pressure: one can acquire statistically converged datasets much more rapidly than with conventional $(10-\mathrm{Hz})$ measurement systems. Despite the 1-second measurement duration, we were able to acquire well-converged, phase-averaged datasets that revealed important insight into the structure and dynamics of the flame. Although it is theoretically possible to acquire similarly converged, phase-averaged statistics using conventional $(10-\mathrm{Hz})$ measurement systems, the rapid fouling of the windows by PIV seed particles would make this highly impractical for more than a select few run conditions. High-bandwidth laser imaging diagnostics, therefore, constitute an important enabling technology for the study of flames at high pressure, rendering otherwise unfeasible measurements both practical and affordable.

\section{Conclusion}

In this study, high-bandwidth, laser-based imaging diagnostics were applied to study the structure and dynamics of a series of turbulent, swirl-stabilized flames at equivalence ratios ranging from $\phi=0.45$ to 1.2 , and pressures ranging from 3 to 7 bars. Stereo-PIV measurements, acquired at $9.3 \mathrm{kHz}$ over periods of approximately 1 second were used to characterize the flow-field near the exit plane of the combustor. OH-PLIF measurements were acquired synchronously with the PIV at a rate of $3.1 \mathrm{kHz}$. Gradient-filtering was used to identify reaction zones in the images and thereby characterize the mean flame surface density.

Comparison of the mean velocity and FSD fields showed that whereas increasing pressure had little effect on the flame structure, variation of the stoichiometry from lean to rich resulted in a major change of flame structure and dynamics. The high bandwidth of these measurements enabled us to perform a phase averaging of the data with respect to the dominant flow-field oscillation (identified via proper orthogonal decomposition). This showed that at lean conditions, the dominant flow-field oscillation is an axial forcing associated with thermoacoustic pulsation. At rich conditions, the dominant oscillation was associated with a precessing vortex core in the inner recirculation zone. These results are consistent with prior measurements in a burner with similar geometry at atmospheric pressure.

\section{References}

\footnotetext{
${ }^{1}$ O. Lammel, K.P. Geigle, R. Lückerath, W. Meier, M. Aigner, GT2007-27902, Proc. ASME Turbo Expo 2007, (2007) Montreal (Canada).

${ }^{2}$ P. Weigand, W. Meier, X.R. Duan, W. Stricker, M. Aigner. “ Investigations of swirl flames in a gas turbine model combustor. I. Flow field, structures, temperature, and species distributions" Comb. Flame 144:1-2 (2006), 205-224.

${ }^{3}$ K.P. Geigle, J. Zerbs, M. Köhler, M. Stöhr, W. Meier. "Experimental Analysis of Soot Formation and Oxidation in a Gas Turbine Model Combustor Using Laser Diagnostics". J Eng Gas Turb Power 133 (2011) 121503-1.

${ }^{4}$ K.P. Geigle, R. Hadef, W. Meier. "Soot Formation and Flame Characterization of an Aero-Engine Model Combustor Burning Ethylene at Elevated Pressure", J. Eng. Gas Turbines Power 136 (2014) 021505.

${ }^{5}$ K.P. Geigle, M. Köhler, W. O'Loughlin, W. Meier. "Investigation of soot formation in pressurized swirl flames by laser measurements of temperature, flame structures and soot concentrations", Proc. Comb. Inst. 35 (2015) 3373-3380.

${ }^{6}$ Boxx, I., Stöhr, M., Carter, C., Meier, W., "Temporally Resolved Planar Measurements of Transient Phenomena in a Partially Pre-mixed Swirl Flame in a Gas Turbine Model Combustor”, Combust. Flame 157 (2010), 1510-1525.

${ }^{7}$ Boxx, I., C. Slabaugh, P. Kutne, R.P. Lucht, W. Meier. "3kHz PIV / OH-PLIF measurements in a gas turbine combustor at elevated pressure", Proc. Combust. Inst. 35 (2014). 3793-3802.

${ }^{8}$ R. Sadanandan, M. Stöhr, W. Meier, "Simultaneous OH-PLIF and PIV measurements in a gas turbine model combustor", Appl. Phys. B 90 (2008) 609-618.

${ }^{9}$ C.D. Slabaugh, I. Boxx, S. Werner, R.P. Lucht, W. Meier. "Temporally-Resolved Measurements of the Structure and Dynamics in Partially-Premixed Swirl Flames at Elevated Power Density”, AIAA J. (2015. Accepted).

${ }^{10}$ G. Berkooz, P. Holmes, J.L. Lumley, "The Proper Orthogonal Decomposition In The Analysis Of Turbulent Flows", Ann. Rev. Fluid Mech., (1993) 539-575.
} 
${ }^{11}$ L. Sirovich. "Turbulence and the dynamics of coherent structures, Part I: Coherent structures", Q. Applied Mathematics. XLV:3 (1987) 561-571.

${ }^{12}$ P. Weigand, W. Meier, X.R. Duan, R. Giezendanner-Thoben, U. Meier, “'Laser Diagnostic Study of the Mechanism of a Periodic Combustion Instability in a Gas Turbine Model Combustor", Flow Turbul. Combust. 75 (2005) 275-292.

\section{Tables}

Table 1 - Burner Operating Conditions

\begin{tabular}{|c|c|c|c|c|}
\hline Condition & Pressure (Bars) & Stoichiometry & $\begin{array}{c}\text { Thermal Load } \\
(\mathrm{kW})\end{array}$ & Oxidation Air? \\
\hline 1 & 3 & 0.67 & 21.6 & No \\
\hline 2 & 5 & 0.67 & 35.9 & No \\
\hline 3 & 7 & 0.67 & 50.3 & No \\
\hline 4 & 5 & 0.45 & 24.2 & No \\
\hline 5 & 5 & 1.2 & 64.4 & Yes \\
\hline
\end{tabular}

Table 2 - Peak OH-PLIF SNR vs. Pressure

\begin{tabular}{|c|c|}
\hline $\begin{array}{c}\text { Pressure } \\
\text { (bars) }\end{array}$ & Peak SNR \\
\hline 3 & 48 \\
\hline 5 & 43 \\
\hline 7 & 37 \\
\hline
\end{tabular}




\section{Figures}

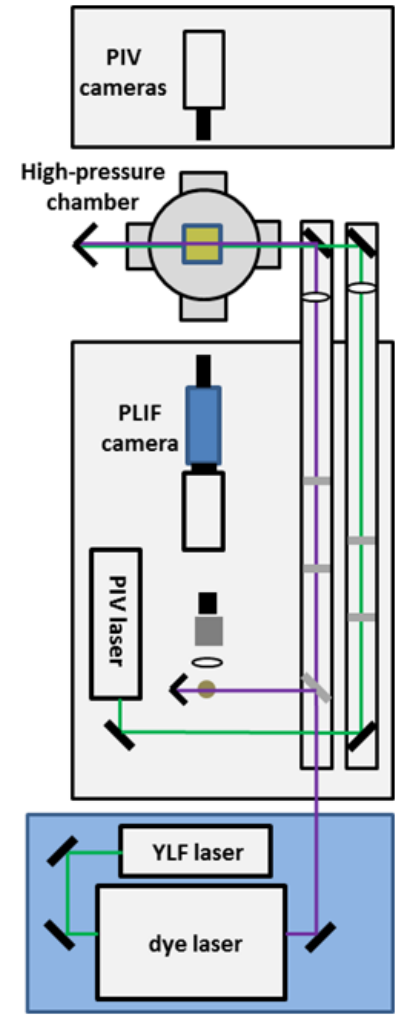

Figure 1 - Experimental Setup
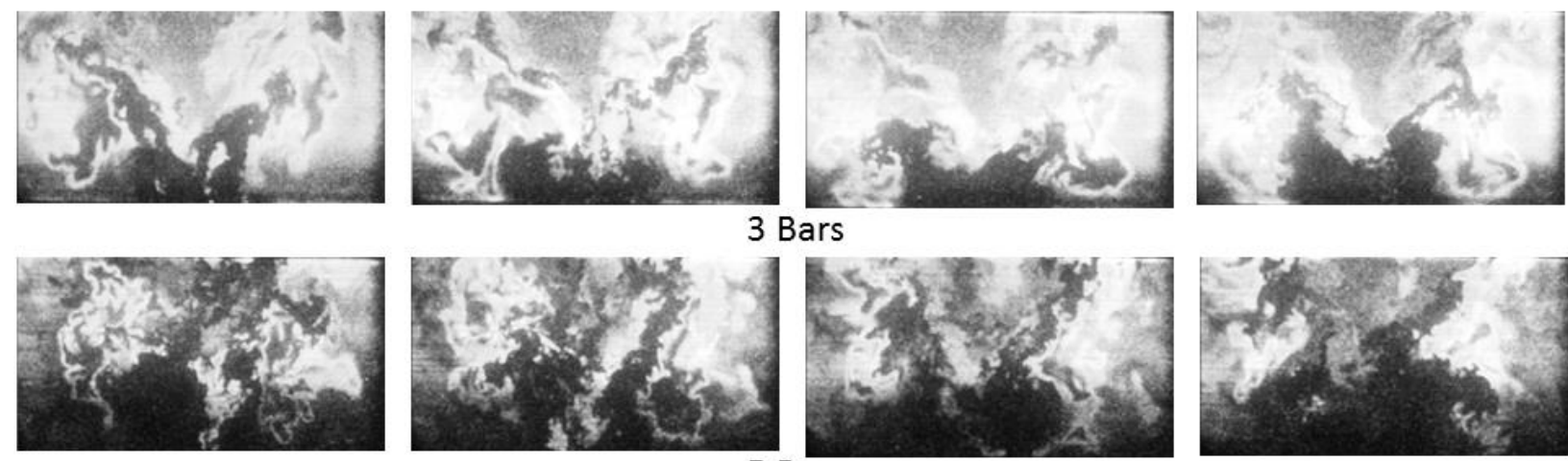

3 Bars
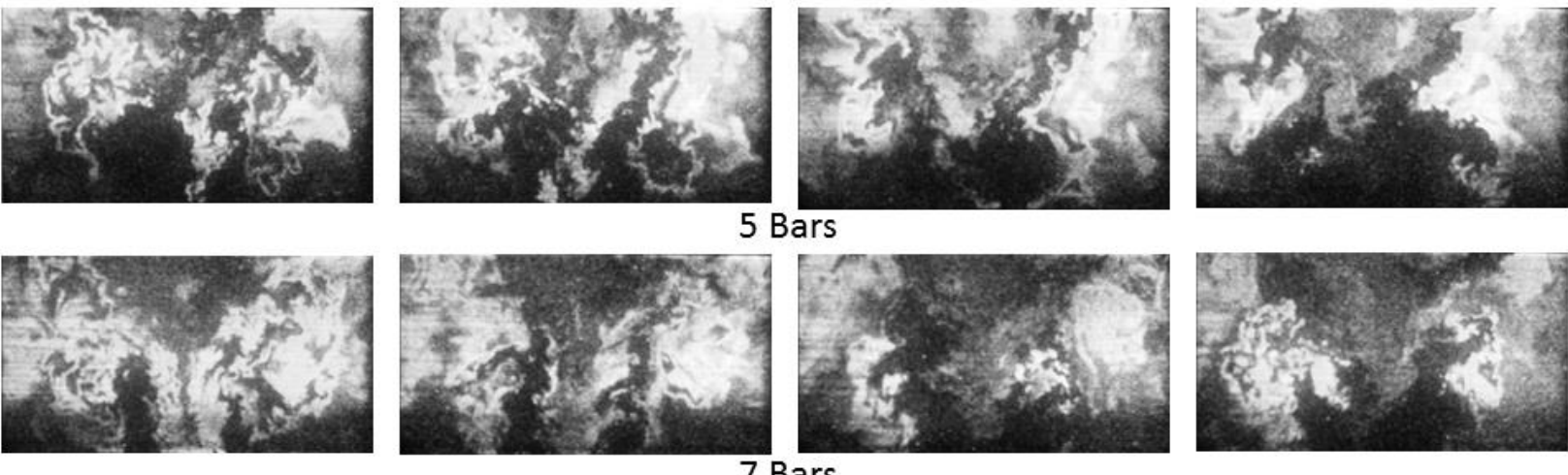

7 Bars

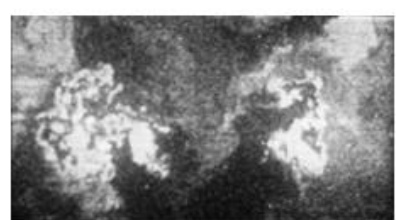

Figure 2 - Sample OH-PLIF image sequences acquired at $3.1 \mathrm{kHz}$ in swirl-stabilized, ethylene-air flames at stoichiometry $\phi=0.67$ and increasing chamber pressure. Note, all colorbars are identical, to emphasize the decreasing signal-to-noise ratio with increasing chamber pressure. 

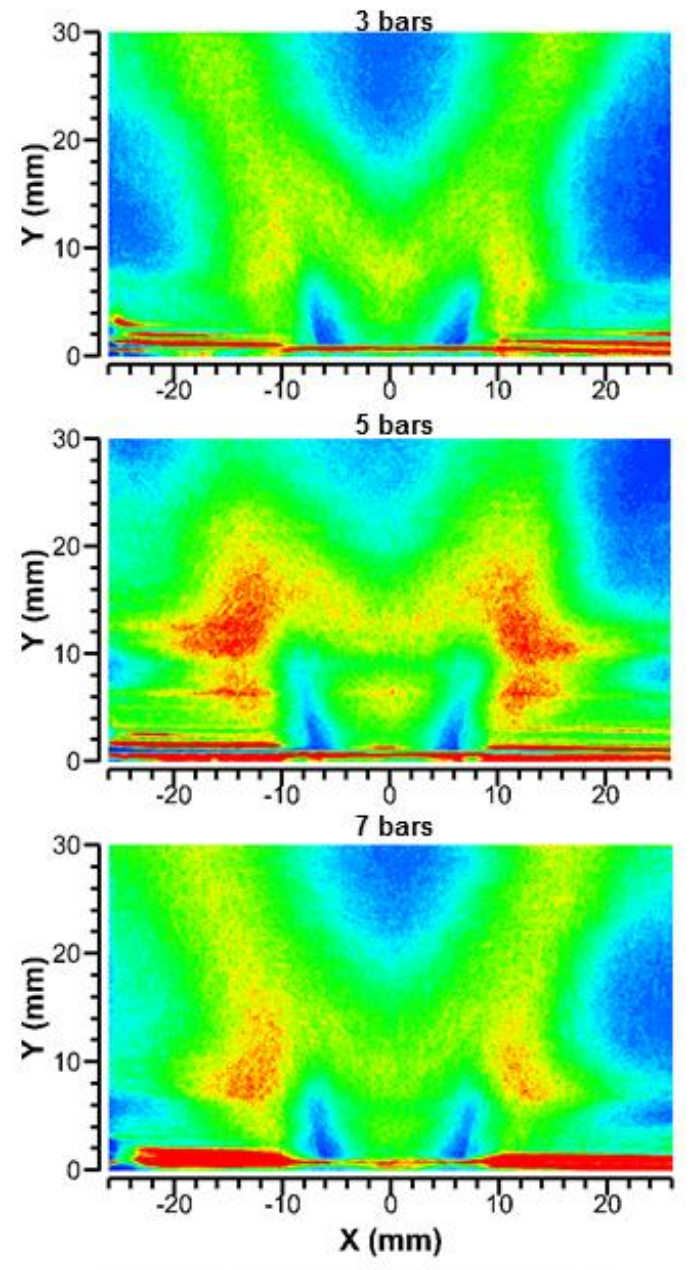

Figure 3 - Mean Flame Surface Density vs. Pressure
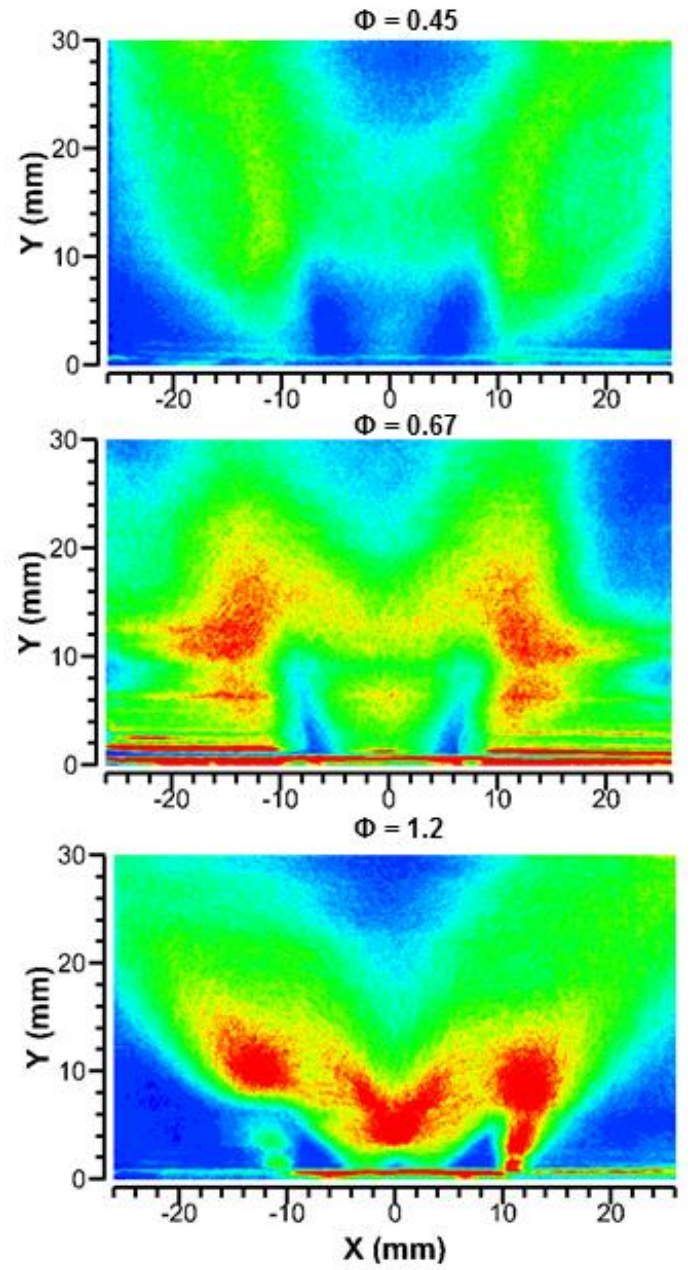

Figure 4 - Mean Flame Surface Density vs. Stoichiometry 

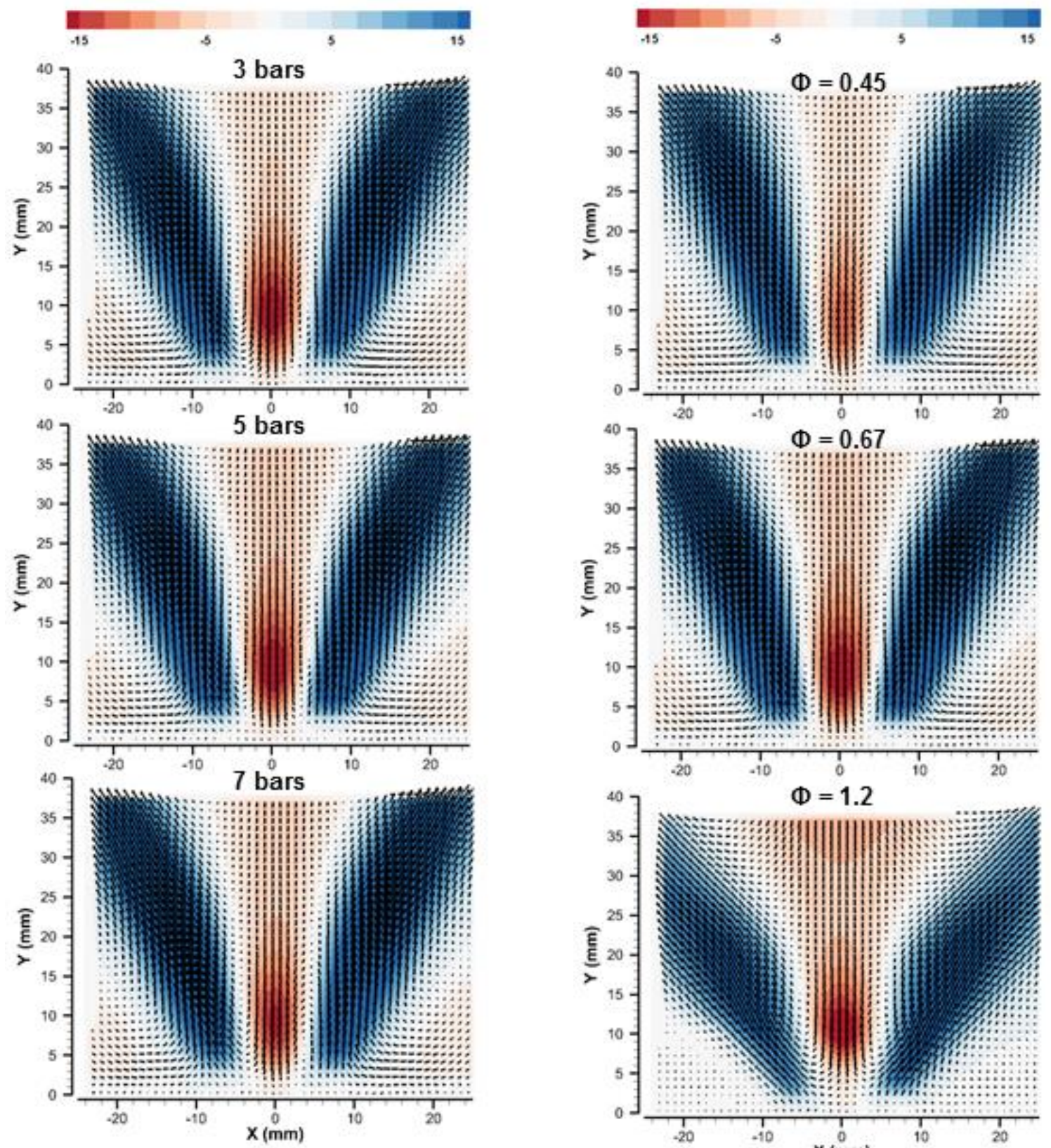

Figure 5 - Mean axial velocity $(\mathrm{m} / \mathrm{s}$ ) vs. pressure

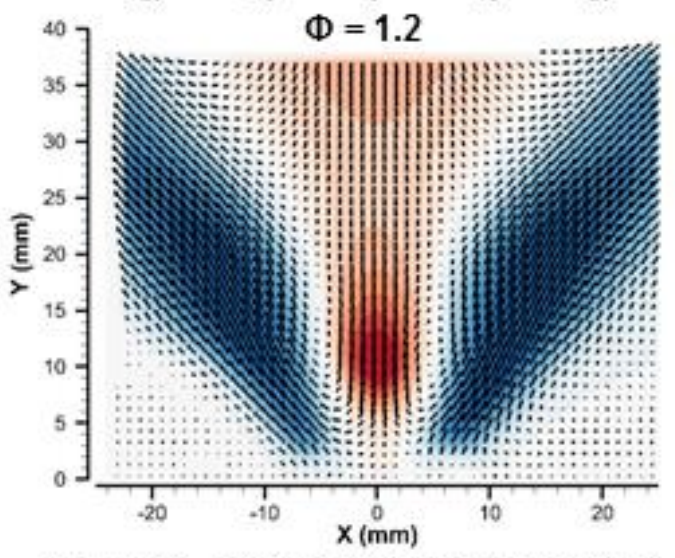

Figure 6 - Mean axial velocity $(\mathrm{m} / \mathrm{s})$ vs. stoichiometry 
Phase 1
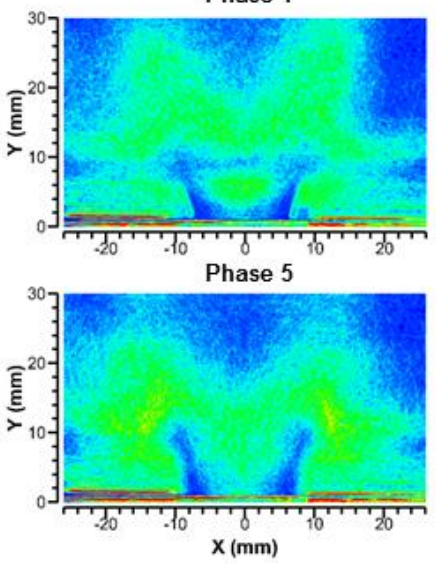

Phase 2
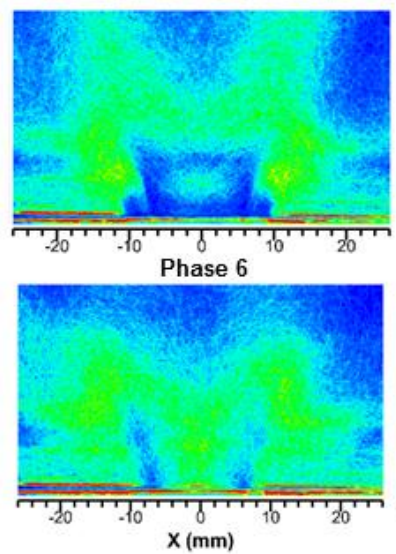

Phase 3

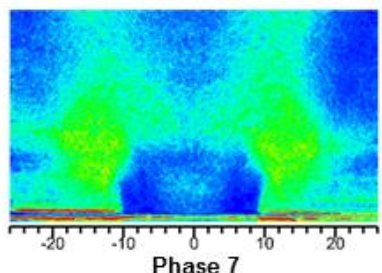

Phase 7

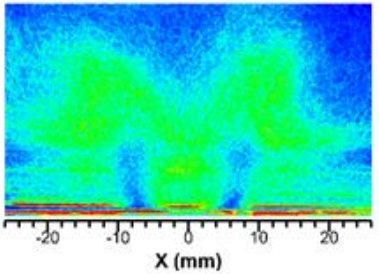

Phase 4
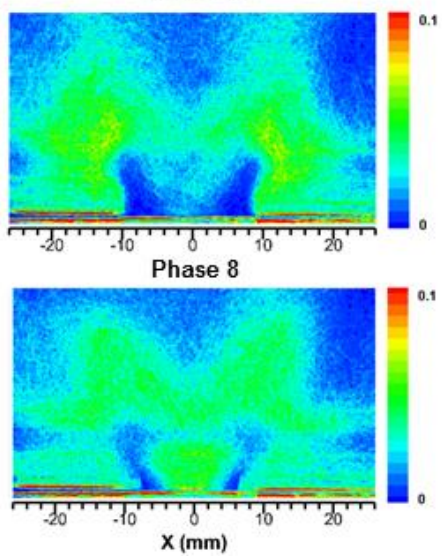

Figure 7 - Mean flame surface density, phase averaged with respect to the first POD mode. $P=5$ bars, $\Phi=0.67$.

Phase 1

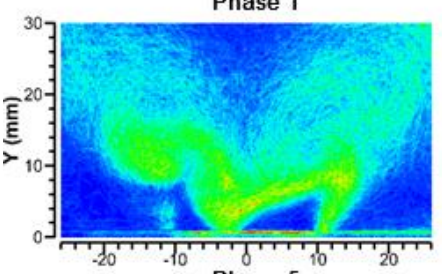

Phase 5

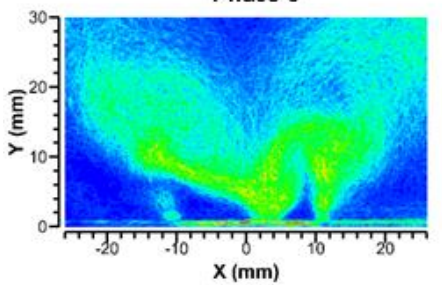

Phase 2

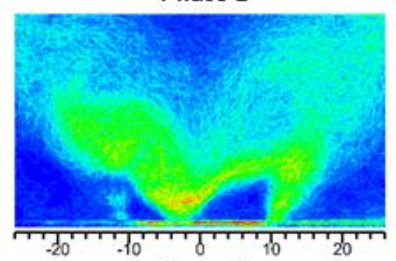

Phase 6

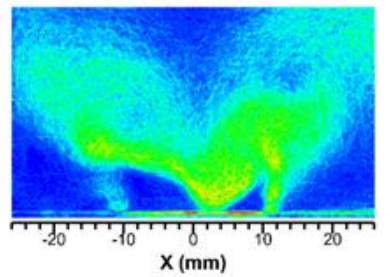

Phase 3

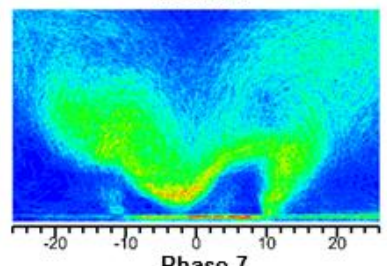

Phase 7

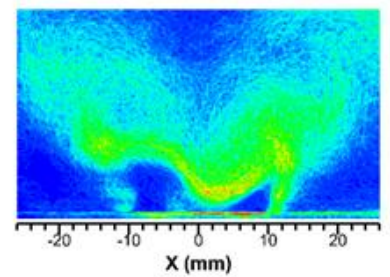

Phase 4

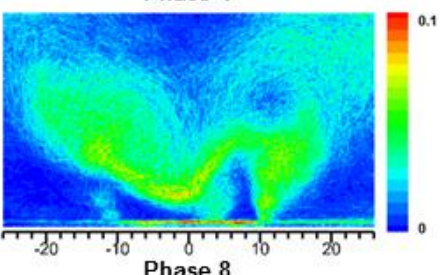

Phase 8

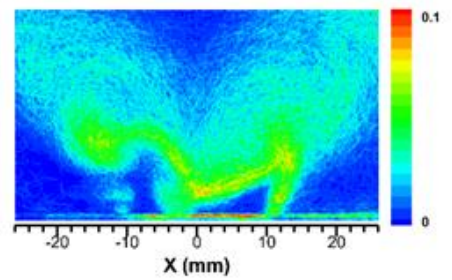

Figure 8 - Mean flame surface density, phase averaged with respect to the first POD mode. $P=5$ bars, $\Phi=1.2$ 